\title{
Algorithms of Relationships between Left Ventricle Dysfunction and Atrial Fibrillation
}

\section{Vladimir Tilman*}

The Sheba Medical Center, Rehabilitation Hospital, Ramat Gan, Israel

\begin{abstract}
Atrial fibrillation (AF) is the most common cardiac arrhythmia. Many studies have investigated the cause for the development of $A F$ in the left ventricle dysfunction. Recently the hypothesis was proposed that atrial fibrillation is a protective physiological mechanism, based on the termination of atrial mechanical systole. This reduces the enddiastolic pressure in the left ventricle by excluding the component of systolic left atrial pressure from the total enddiastolic pressure and, respectively, in the system of the pulmonary veins and alveolar capillaries in situation of the left ventricle dysfunction, and thus reduces the likelihood for development of pulmonary congestion. First of all this hypothesis explains relationship between left ventricle dysfunction and AF. The hypothesis helps to build algorithms of the development $\mathrm{AF}$ in the cases of the left ventricle dysfunction in the various diseases and conditions.
\end{abstract}

Keywords: Atrial fibrillation; Left ventricle dysfunction; Enddiastolic pressure; Algorithms

\section{Introduction}

Atrial fibrillation (AF) is the most common cardiac arrhythmia in clinical practice and is most often associated with left ventricle (LV) dysfunction/heart failure (HF). The prevalence of AF in patients with heart failure increases in parallel with the severity of the disease, ranging from $5 \%$ in patients with mild, from $10 \%$ to $26 \%$ among patients with moderate, and up to $50 \%$ in patients with severe heart failure [1]. Many studies have investigated the cause for the development of AF, however, the question remains unanswered. The causative relationship between the two conditions has not been fully determined. Many studies on the subject: "AF - a cause or consequence?" have been carried out, however the question remain unanswered. Last year the hypothesis was proposed, that atrial fibrillation acts as a protective pathophysiological mechanism, based on the termination of atrial mechanical systole $[2,3]$. The reasoning of the hypothesis is as follows. The left atrial (LA) systole begins $0.12-0.2 \mathrm{~s}$ before the ending of the LV diastole, thus compressing the atrial volume and increasing its internal pressure. An algebraic addition therefore occurs by combining the "positive" pressure of LA systole (atrial kick) with the "negative" pressure of the LV diastole, resulting in the end-diastolic pressure (EDP). At the end of the LV diastole, while the MV is still open, the EDP is immediately transmitted and equalized through the communicating system of the LV, LA, pulmonary veins (PV), and alveolar capillaries (AC), as there are no obstacles to spread of the EDP wave. EDP is thus equal to pulmonary wedge pressure (PWP), with a normal range of $\sim 8-12$ $\mathrm{mm} \mathrm{Hg}$.

AF stops mechanical systole of the LA excluding the component of systolic LA pressure from total pressure in the LA-PV-AC system, thus leading to reduced EDP and PWP. The right atrium (RA) is also involved in AF. As is in the case with the LA, there is a termination of the mechanical systole of the right atrium (RA), and a reduction in enddiastolic pressure in the right ventricle (RV). Thus at the end of diastole of the RV when the tricuspid valve is still open, pressure of systemic venous inflow to the heart is decreased, resulting in a reduction of the preload. Thus, AF leads to a synergy in the hydrodynamic effect, due to the termination of a mechanical systole in both the LA and RA. This reduces the pressure in the system of the pulmonary veins and alveolar capillaries in pathological situations, and thus reduces the likelihood for development of pulmonary congestion and edema. The hypothesis is well correlated with the known facts and phenomenon's associated with $\mathrm{AF}$, and explains the causes of the onset of AF in different conditions and diseases (Table 1).

It is known that the main manifestation of LV dysfunction (systolic and diastolic) is a significant increase of EDP which is transmitted through the entire intercommunicated system (LV-LA-PV-AC), causing increased PWP with increased likelihood for development of pulmonary congestion and edema. Pulmonary edema is usually present when PWP increases to $20 \mathrm{~mm} \mathrm{Hg}$. It is logical to assume that the termination of both left and right side mechanical atrial systole, as a result of the development of the AF, creates more favorable, more optimal hydrodynamic conditions for the heart in a status of LV dysfunction, which is a manifestation of many cardiovascular diseases.

This HYPOTHESIS was proposed that AF acts as the physiological protective mechanism. The main purpose of $\mathrm{AF}$ is to eliminate the mechanical systole of the LA in order to reduce the intercommunicated pressure in the system (LA-PV-AC), and of the RA with the effect of reducing preload, and thus prevent pulmonary congestion and edema in the conditions and the diseases which cause to increase this pressure. By means of this hypothesis it is possible to present an algorithm of relationships between AF and LV dysfunction (Figures 1-5).

\begin{tabular}{|c|c|}
\hline Sinus Rhythm & Atrial Fibrillation \\
\hline $\mathrm{EDP}=\mathrm{DPlv}+\mathrm{AKp}$ & $\mathrm{EDP}=\mathrm{DPlv}$ \\
\hline
\end{tabular}

EDP - total end diastolic pressure of left ventricle,

DPIv - diastolic pressure of left ventricle at the end of diastole,

$\mathrm{AKp}$ - systolic left atrial pressure (atrial kick pressure).

Table 1: The causes of the onset of AF in different conditions and diseases.

*Corresponding author: Vladimir Tilman, The Sheba Medical Center, Rehabilitation Hospital, Ramat Gan, Israel, Tel: 972-0547345525; E-mail: water5945@gmail.com

Received June 07, 2016; Accepted June 24, 2016; Published June 30, 2016

Citation: Tilman V (2016) Algorithms of Relationships between Left Ventricle Dysfunction and Atrial Fibrillation. J Cardiovasc Dis Diagn 4: 247. doi: 10.4172/23299517.1000247

Copyright: ( $) 2016$ Tilman V. This is an open-access article distributed under the terms of the Creative Commons Attribution License, which permits unrestricted use, distribution, and reproduction in any medium, provided the original author and source are credited. 


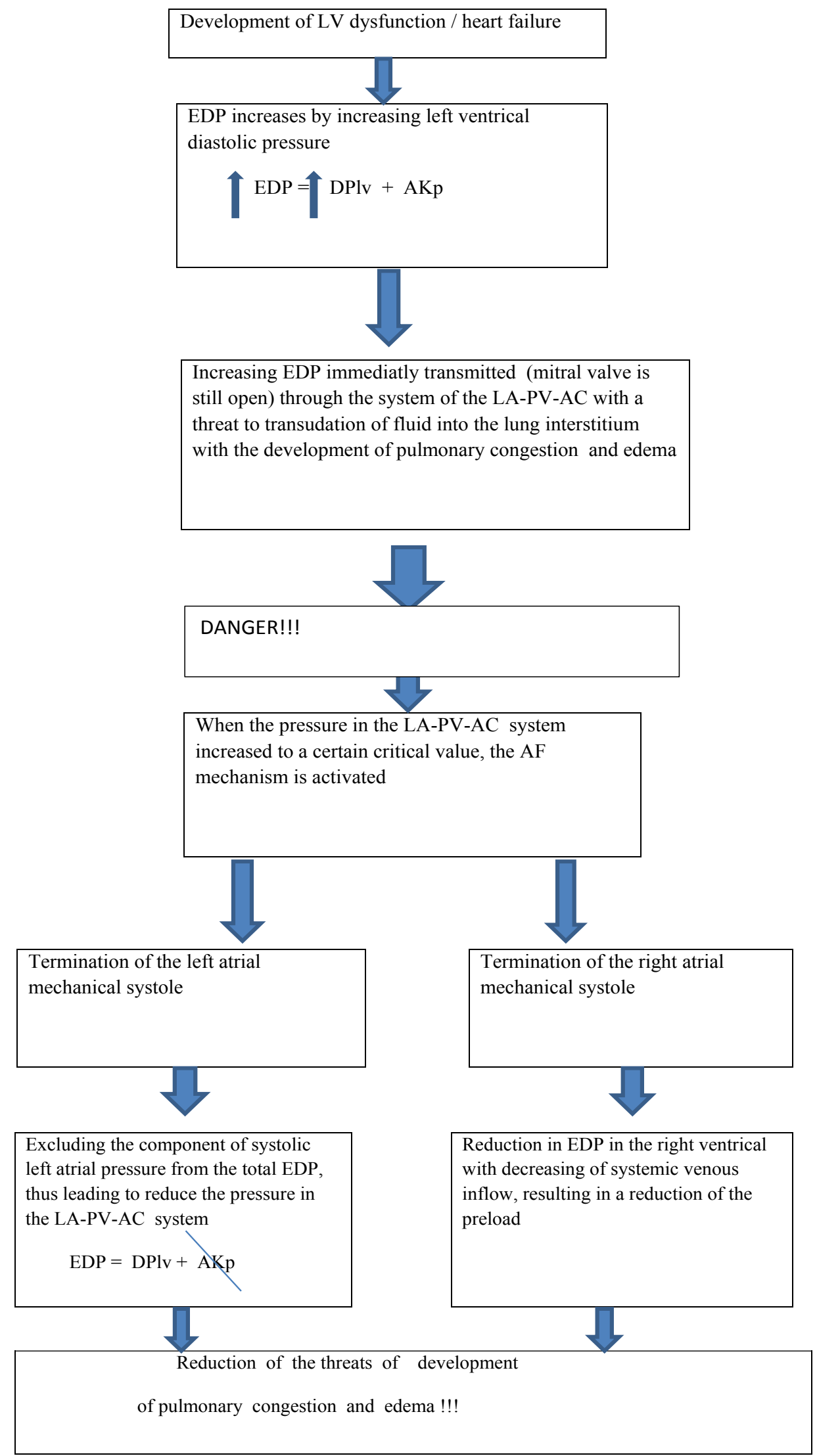

Figure 1: LV dysfunction and AF - algorithm of relationship. 
Citation: Tilman V (2016) Algorithms of Relationships between Left Ventricle Dysfunction and Atrial Fibrillation. J Cardiovasc Dis Diagn 4: 247. doi: 10.4172/2329-9517.1000247

Similary, we can imagine algorithms of the development AF in various diseases [4-6].

1. Hypertension and AF- algorithm of relationship

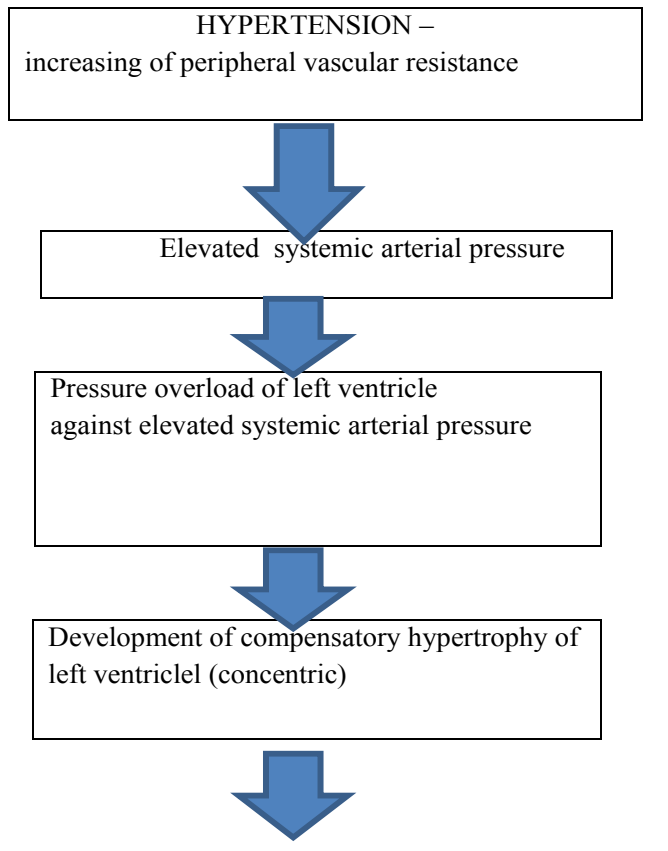

Development of diastolic dysfunction with

decrease of relaxation function

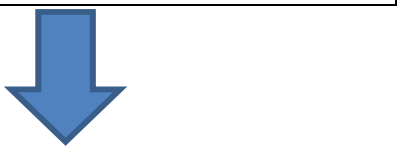

EDP increases by increasing left ventricle diastolic pressure

1 $\mathrm{EDP}=\uparrow \mathrm{DPlv}+\mathrm{AKp}$

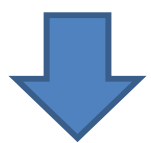

Increasing EDP immediatly transmitted (mitral valve is still open) through the system of the LAPV-AC with a threat to transudation of fluid into the lung interstitium with the development of pulmonary congestion and edema

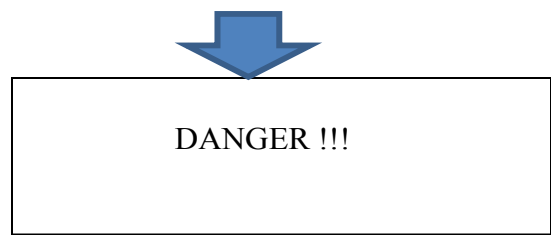




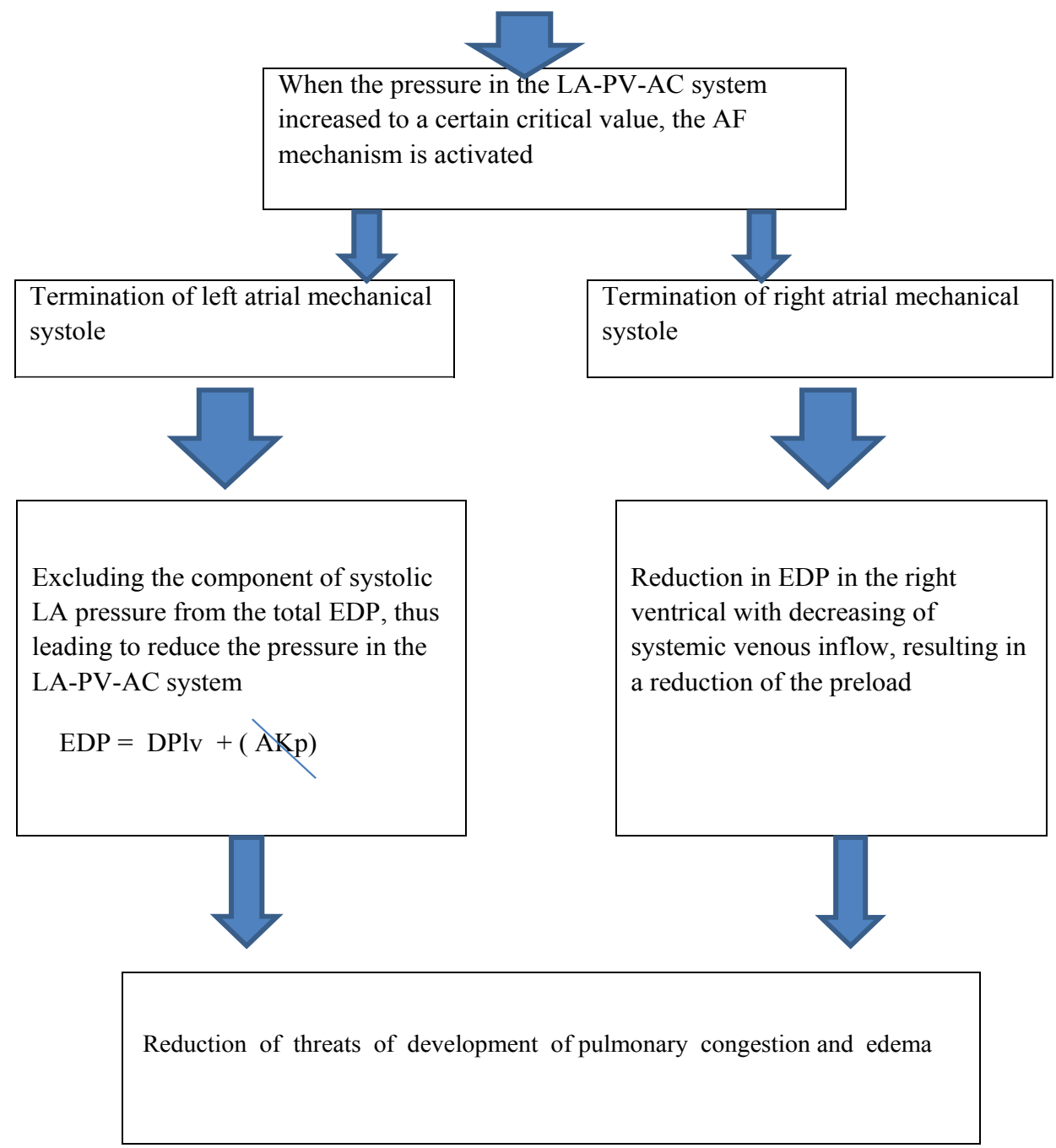

Figure 2: Hypertention and AF.

MITRAL STENOSIS -
obstruction to LV inflow at the level of the mitral valve

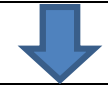

The resting diastolic mitral valve gradient, and hence LA pressure, increases
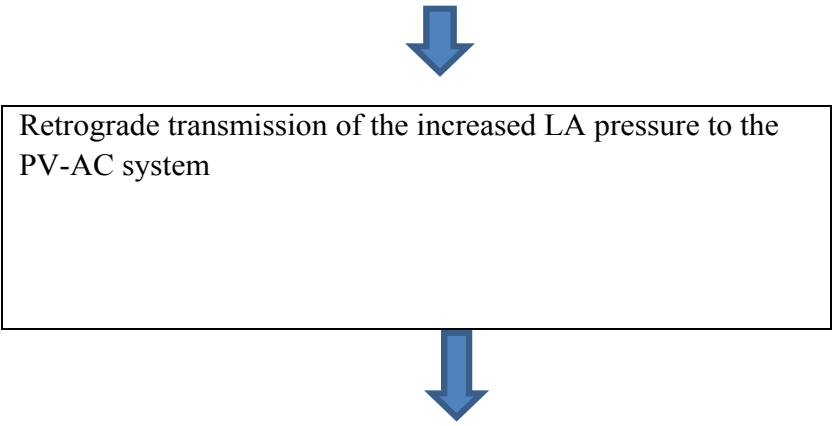
Citation: Tilman V (2016) Algorithms of Relationships between Left Ventricle Dysfunction and Atrial Fibrillation. J Cardiovasc Dis Diagn 4: 247. doi: 10.4172/2329-9517.1000247

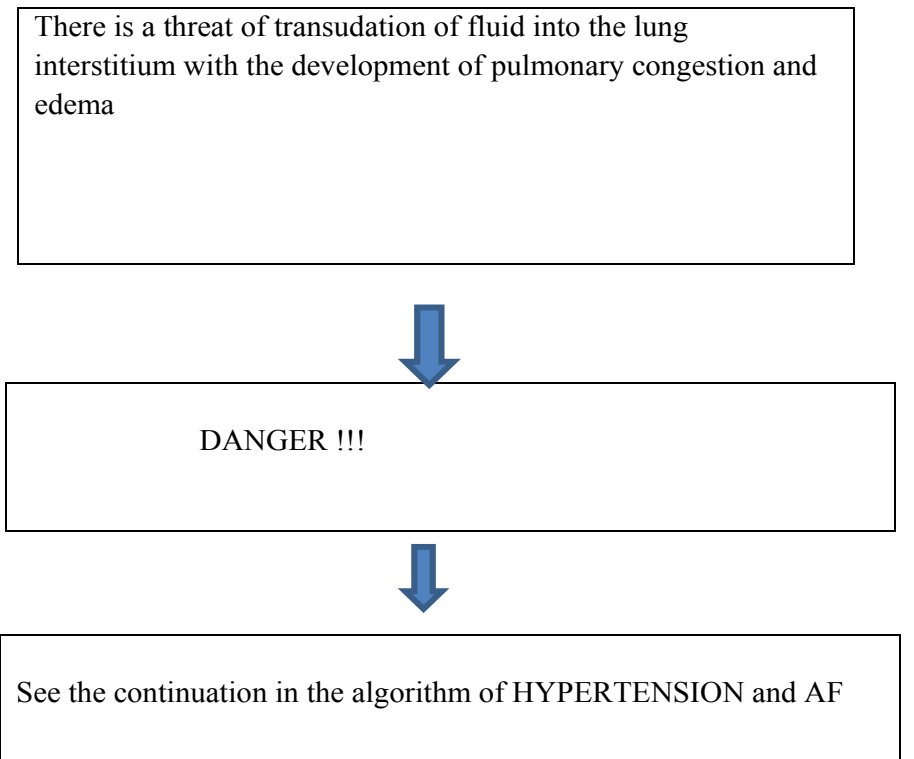

Figure 3: Mitral stenosis and AF.

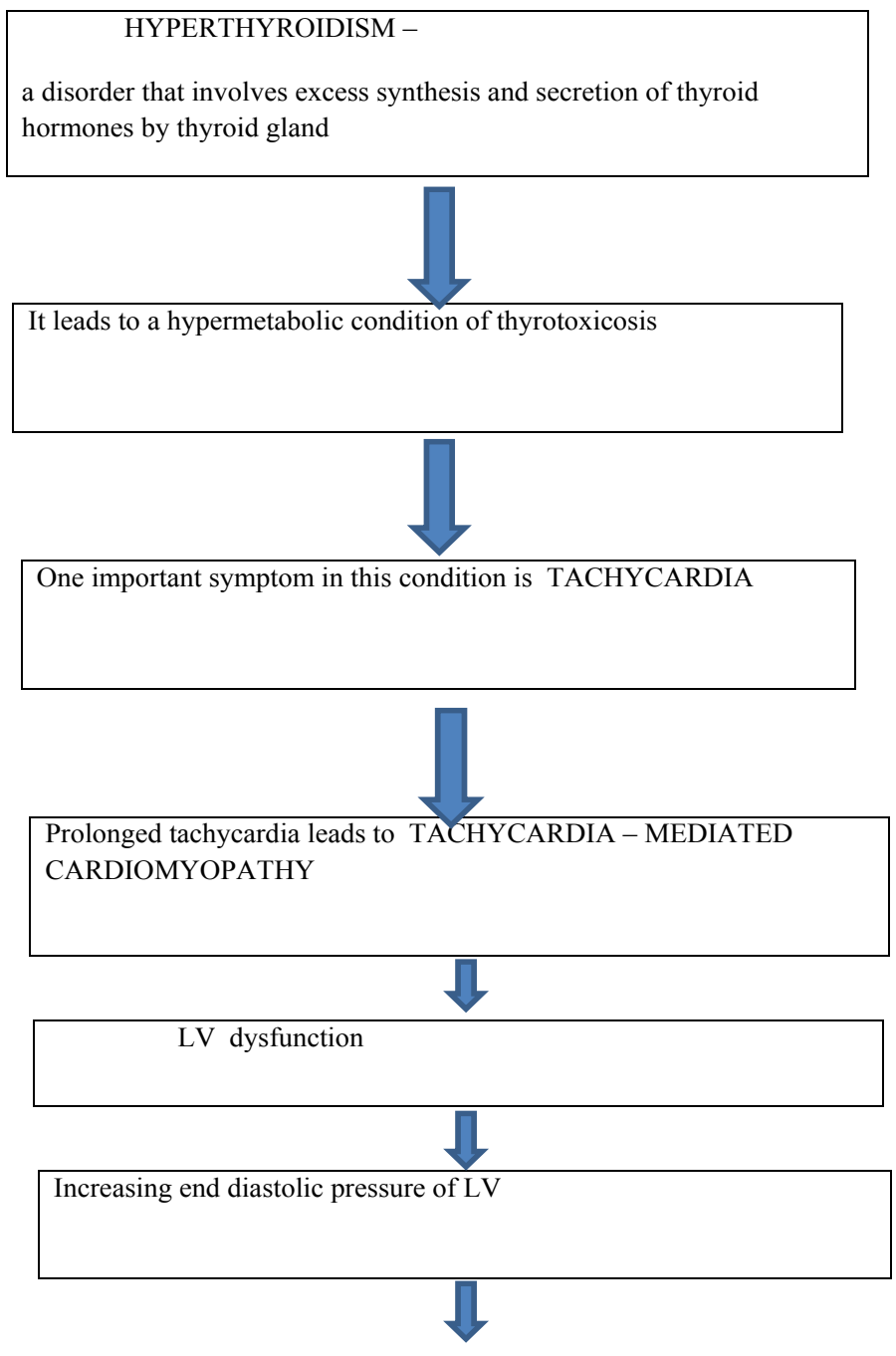


Citation: Tilman V (2016) Algorithms of Relationships between Left Ventricle Dysfunction and Atrial Fibrillation. J Cardiovasc Dis Diagn 4: 247. doi: $10.4172 / 2329-9517.1000247$

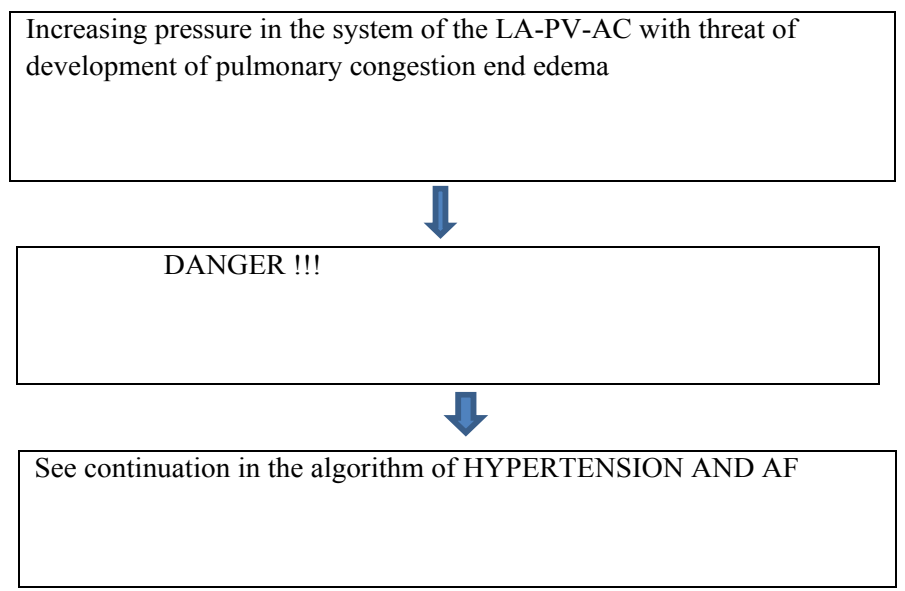

Note: Similary, we can build a relationship algorithm of other diseases and conditions, accompanied by an increase in EDP, with the occurrence of AF.

Figure 4: Hyperthyroidism and AF.

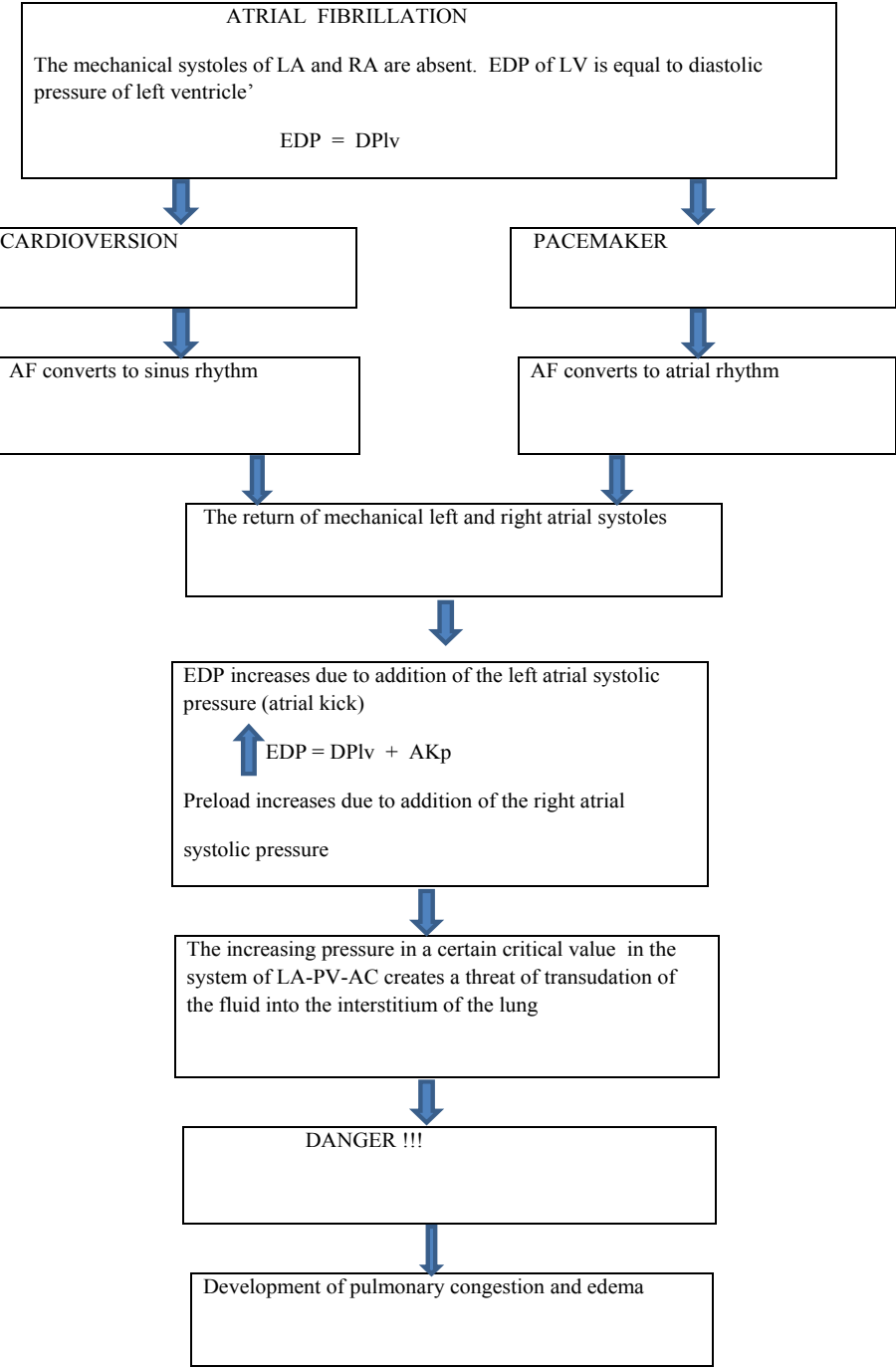

Figure 5: The literature describes cases from medical practice of worsening of the patient's conditions with pulmonary congestion or edema after cardioversion from $\mathrm{AF}$ to synus rhythm or after implantation of the pacemaker (atrial rhythm). The proposed hypothesis can explain this complication and build algorithm of this phenomenon $[7,8]$. 
Citation: Tilman V (2016) Algorithms of Relationships between Left Ventricle Dysfunction and Atrial Fibrillation. J Cardiovasc Dis Diagn 4: 247. doi: $10.4172 / 2329-9517.1000247$

\section{Conclusion}

The above proposed hypothesis suggested that $\mathrm{AF}$ is a physiological protective mechanism for reducing the pressure in the LA-PV-AC system by terminating the atrial mechanical systoles. This hypothesis clearly explains many facts and phenomenon associated with AF and allows the building of algorithms showing relationships between $\mathrm{LV}$ dysfunction and AF, different diseases and conditions and AF. Also it can help to understand the pathophysiological mechanism of development of AF.

\section{References}

1. Maisel WH, Stevenson LW (2003) Atrial fibrillation in heart failure: epidemiology, pathophysiology, and rationale for therapy. Am J Cardiol 91: 2D-8D.

2. Tilman V (2014) Atrial fibrillation and heart failure: is atrial fibrillation a disease? Med Hypotheses 83: 299-301.
3. Tilman V (2016) Atrial fibrillation: In the light of new hypothesis. Med Hypotheses 86: 10-13.

4. Aksnes TA, Kjeldsen SE (2010) A link between hypertension and atria fibrillation: methods of treatment and prevention. Curr Vasc Pharmacol 8 : 769-774.

5. Karthikeyan G, Ananthakrishnan R, Devasenapathy N, Narang R, Yadav R, et al. (2014) Transient, subclinical atrial fibrillation and risk of systemic embolism in patients with rheumatic mitral stenosis in sinus rhythm. Am J Cardiol 114: 869-874.

6. Frost $L$, Vestergaard $P$, Mosekilde $L$ (2004) Hyperthyroidism and risk of atrial fibrillation or flutter: a population-based study. Arch Intern Med 164: 1675-1678.

7. Gowda RM, Misra D, Khan IA, Schweitzer P (2003) Acute pulmonary edema after successful electrical cardioversion of atrial fibrillation. Am J Ther 10 : 73-74.

8. Ellenbogen KA, Gilligan DM, Wood MA, Morillo C, Barold SS (1997) The pacemaker syndrome -- a matter of definition. Am J Cardiol 79: 1226-1229. 